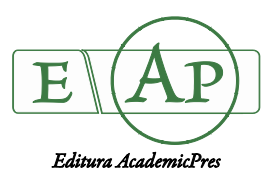

\title{
Chemical Compositions and Sugar Profiles of Consumed Chestnut Cultivars in the Marmara Region, Turkey
}

\author{
Cevriye MERT*, Ümran ERTÜRK \\ Uludag University, Faculty of Agriculture, Department of Horticulture, Görükle Campus 16059, Bursa, \\ Turkey; cevmert@uludag.edu.tr (*orrespondingauthor);umrane@uludag.edu.tr
}

\begin{abstract}
In this study, the chemical compositions and sugar profiles of seventeen local cultivars and two foreign chestnut hybrids, grown in the Marmara Region, Turkey, were examined. The results showed that chestnut hybrids and cultivars have 58.1269.83 total carbohydrates, 10.59-22.38 total sugars, 2.41-3.41 invert sugar, 6.15-12.44 total protein, 2.09-4.36 ash and 0.872.61 total fat values ( $\left(\mathrm{g} 100 \mathrm{~g}^{-1}\right.$ dry matter basis). It was determined that chestnut cultivars generally have over $50 \%$ water content and higher starch content $\left(40.99-53.16 \mathrm{~g} 100 \mathrm{~g}^{-1}\right)$. The sucrose contents of the cultivars were higher than the other sugars. Sucrose, glucose and fructose contents were 10.77-21.66, 0.33-1.13, and 0.15-0.79, respectively (g $100 \mathrm{~g} \mathrm{~g}^{-1}$ dry matter basis). These results stated that chestnuts have rich nutritive substances for human nutrition and health.
\end{abstract}

Keywords: Castanea sativa, glucose, fructose, nutritional composition, sucrose

\section{Introduction}

Turkey has one of the most important and largest productions of chestnut in the world. The naturally spreading chestnut species in Turkey is the European chestnut (Castanea sativa Mill.), and chestnut production utilizes native cultivars. Most chestnut production in Turkey is in the Marmara, Aegean and Black Sea Regions (Soylu et al., 2009; Soylu and Mert, 2009). Chestnut can be consume fresh by roasting and boiling in Turkey, is used in making cakes and is widely used in the candy industry (Uylaser et al., 2014).

Chestnuts have played an important role in human nutrition since ancient times. The term "bread tree" has been used in some places for chestnuts (Bounous et al., 2000). Chestnuts contain high carbohydrates, protein and dietary fibre. In addition, chestnuts differ from other nuts for their low fat and salt content, which make them ideally suited for human nutrition and health (Mujić et al., 2010). Carbohydrates are the relevant components in chestnut, especially starch, followed by sucrose. Together with sucrose, glucose, fructose and raffinose are present in significant amounts and may contribute to the identification of a specific chestnut cultivar (Bernárdez et al., 2004; De la Montana Miguelez et al., 2004; Barreira et al., 2010; Warmund et al., 2011; Suarez et al., 2012). Due to the large proportion of moisture, sugar content, enzyme activity and pericarp characteristics, the shelf life of chestnuts is very limited (Correia et al., 2009). According to several studies (De La Montana Miguelez et al., 2004; Pereira-Lorenzo et al., 2006;
Ertan, 2007; Borges et al., 2008; Silvanini et al., 2014; Yang et al., 2015; Poljak et al., 2016), the chemical composition of chestnut fruits can be changed by cultivar (genotype), environmental factors (climatic conditions, soil characteristics and production practices) and altitude.

The aim of this study was to investigate the chemical composition and sugar profiles of grafted chestnut cultivars/genotypes that are commonly cultivated in the Marmara Region, Turkey.

\section{Materials and Methods}

\section{Fruitsamples}

The seventeen native chestnut cultivars (cvs. 'Akkozak', 'Alimolla', 'Demirci', 'Dursun', 'Firdola', 'Hacibiș, 'Haciömer', 'Halilibrahim', 'İnegölkestanesi', 'İzmitli', 'Karamehmet', 'Korucu', 'Osmanoğlu', 'Öküzgözü', 'Sarıaşlama', 'Sarkestane', and 'Tepeköysarısi') two foreign hybrids (cvs. 'Maraval' and 'Marigoule'), were collected from important place in term of growing chestnuts in the Marmara Region. The fruits were harvested from 25 to 40 -year-old trees from the beginning of September until the end of October.

\section{Determination of chemical compositions}

The moisture content of the chestnuts was determined by the gravimetric method using a drying oven at $105 \pm 2{ }^{\circ} \mathrm{C}$. Total ash content was measured according to AOAC (2000). The total nitrogen was analysed using the Kjeldahl method, and crude protein content was calculated using a nitrogen conversion factor of 5.30, which is specific for chestnut fruits (AOAC, 2000). Total fat was determined after extraction with ether for $16 \mathrm{~h}$ in a 
204

soxhlet device (AOAC, 2000). The dinitrophenol method was utilized in the analysis of total carbohydrates, total sugar and invert sugar (Ross, 1959) using the Beckman Du 530 model spectrophotometer. Starch quantity was calculated by multiplying the value obtained via subtracting the total sugars from the total carbohydrates by the coefficient 0.94 .

\section{Sugar Analysis}

\section{Extraction of sugar using and ultrasound bath}

The fruit samples were carried to the laboratory, peeled, and stored at $-20^{\circ} \mathrm{C}$ until analysis. Approximately $10-15 \mathrm{~g}$ of frozen homogenized chestnut sample was directly weighed in a $100 \mathrm{ml}$ volumetric flask, $70 \mathrm{ml}$ deionized distilled water was added, and the dissolution was mixed with a magnetic blender for 30 minutes at $70{ }^{\circ} \mathrm{C}$. Afterwards, the $100 \mathrm{ml}$ dissolution was held in an ultrasound bath in $15 \mathrm{~min}$ at $50^{\circ} \mathrm{C}$ and was centrifuged for 10 $\min$ at $3000 \mathrm{~g}$. A millilitre of the dissolution was passed through a $0.45 \mu \mathrm{m}$ filter GHP (Waters, Millford, MA, USA) prior to HPLC analysis.

Determination of sugars using bigh-performance liquid chromatography (HPLC)

Free sugar profiles were determined by high-performance liquid chromatography coupled to a refraction index detector (HPLC-RI). Acetonitrile/deionized water was used as the mobile phase at 80:20 (v/v), the flow rate was $2 \mathrm{ml} / \mathrm{min}$, the column temperature was $35^{\circ} \mathrm{C}$ and the injection volume was 10 $\mu$ l. The used NH2 column had a $4.6 \mathrm{~mm}$ diameter, was $250 \mathrm{~mm}$ long, and had a $5 u \mathrm{~m}$ particle size $(250 \times 4.6 \mathrm{~mm} \mathrm{ID})$. The results are expressed in $\mathrm{g}^{100 \mathrm{~g}^{-1}}$ of dried weight, calculated by an internal standard normalization of the chromatographic peak area. Sugar identification was made by comparing the relative retention times of sample peaks with standards.

\section{Statistical analysis}

Means and standard deviations of chestnut composition (except moisture content) were expressed on a dry weight basis due to the different moisture contents of the samples. Statistical comparisons of the mean values were performed using one-way analysis of variance (ANOVA), followed by Duncan's multiple range test $(\mathrm{p}<0.05$ confidence level) using Minitab 17.0 software. A principal component analysis (PCA) was also performed using Minitab 17.0 software.

\section{Results and Discussion}

\section{Proximate analysis}

The nutritional compositions of the nineteen chestnut cultivars are shown in Table 1. Moisture content of cultivars was determined between $46.52 \%$ and $59.47 \%$ and demonstrated significant differences among all cultivars $(\mathrm{p}<$ 0.05 ). The moisture content of chestnuts should not be less than $49 \%$ or over $60 \%$ in order to be able conserve them in a better way (Glushkova et al., 2010). According to PereiraLorenzo et al. (2006), although high moisture content is very important for the fresh market, it causes mould problems during storage and delivery. The moisture content of chestnuts depends on fruit characteristics and ecological conditions such as soil type, summer rainfalls, altitudes and orchard locations (Borges et al., 2008; Neri et al., 2010).

Total carbohydrates varied significantly among cultivars ( $\mathrm{p}$ $<0.05$ ). Total carbohydrate amount of all cultivars ranged

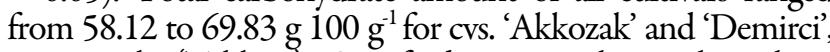
respectively (Table 1). Our findings were lower than those obtained by other researchers. Chestnut fruits generally contain high rates of carbohydrates; the rate was $86.26 \mathrm{~g}^{100 \mathrm{~g}^{-1}}$ in American chestnuts ( $C$. dentata Borkh.) (McCarthy and

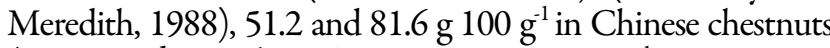
(Yang et al., 2015), and 71.68-88.10 g $100 \mathrm{~g}^{-1}$ in European chestnuts (McCarthy and Meredith, 1988; Künsch et al., 1999; Bounous, 1999; Bounous et al., 2000; Ertürk et al., 2006). This value changed nearly $16 \%$ in the different materials of the C. sativa species (Bounous et al., 2000; Ertürk et al., 2006). The accessions in the study also showed an approximately $12 \%$ variation in carbohydrate content in the highest and lowest cultivars.

The predominant component of dry matter is starch

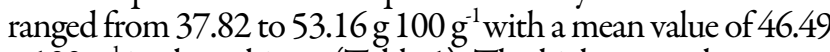
$\mathrm{g} 100 \mathrm{~g}^{-1}$ in the cultivars (Table 1). The highest starch content

Table 1. Proximate composition of the nineteen chestnut cultivars (mean \pm SD)

\begin{tabular}{|c|c|c|c|c|c|c|}
\hline Cultivar & $\begin{array}{c}\text { Moisture } \\
(\%)\end{array}$ & $\begin{array}{l}\text { Carbohydrates } \\
\left(\mathrm{g} / 100 \mathrm{~g}^{-1}\right)\end{array}$ & $\begin{array}{c}\text { Starch } \\
\left(\mathrm{g} / 100 \mathrm{~g}^{-1}\right)\end{array}$ & $\begin{array}{l}\text { Crude protein } \\
\left(\mathrm{g} / 100 \mathrm{~g}^{-1}\right)\end{array}$ & $\begin{array}{l}\text { Crude fat } \\
\left(\mathrm{g} / 100 \mathrm{~g}^{-1}\right)\end{array}$ & $\begin{array}{c}\text { Ash } \\
\left(\mathrm{g} / 100 \mathrm{~g}^{-1}\right) \\
\end{array}$ \\
\hline 'Akkozak' & 53.63 & $58.12 \pm 0.49 c$ & $42.51 \pm 1.79 \mathrm{efg}$ & $8.35 \pm 0.38$ efg & $1.68 \pm 0.05 \mathrm{~cd}$ & $2.59 \pm 0.01 \mathrm{def}$ \\
\hline 'Alimolla' & 55.11 & $68.37 \pm 1.10 \mathrm{ab}$ & $53.16 \pm 0.93 \mathrm{a}$ & $7.46 \pm 1.17 \mathrm{~h} 1$ & $1.65 \pm 0.76 \mathrm{~cd}$ & $4.39 \pm 0.43 \mathrm{a}$ \\
\hline 'Demirci' & 51.18 & $69.83 \pm 3.20 \mathrm{a}$ & $52.90 \pm 2.53 \mathrm{ab}$ & $7.93 \pm 0.15 \mathrm{fgh}$ & $1.10 \pm 0.41 \mathrm{~g}$ & $3.09 \pm 0.50 \mathrm{bcd}$ \\
\hline 'Dursun' & 51.43 & $62.19 \pm 0.79 \mathrm{abc}$ & $45.45 \pm 0.49 \mathrm{def}$ & $12.44 \pm 0.30 \mathrm{a}$ & $1.05 \pm 0.37 \mathrm{~g}$ & $2.09 \pm 0.10 \mathrm{f}$ \\
\hline 'Firdola' & 54.83 & $63.54 \pm 2.03 \mathrm{abc}$ & $43.42 \pm 1.82 \mathrm{def}$ & $7.89 \pm 0.27$ ghı & $1.04 \pm 0.03 \mathrm{~g}$ & $2.91 \pm 0.06 \mathrm{bcd}$ \\
\hline 'Haciibiş' & 49.25 & $68.88 \pm 0.80 \mathrm{ab}$ & $51.45 \pm 1.63 \mathrm{abc}$ & $6.55 \pm 0.38 \mathrm{j}$ & $1.58 \pm 0.03 \mathrm{de}$ & $2.66 \pm 0.21 \mathrm{def}$ \\
\hline 'Hacıömer' & 57.48 & $65.69 \pm 7.37 \mathrm{abc}$ & $49.11 \pm 7.35 \mathrm{a}-\mathrm{d}$ & $7.30 \pm 0.35 \mathrm{~h}$ & $0.92 \pm 0.11 \mathrm{~h}$ & $2.74 \pm 0.45 \mathrm{de}$ \\
\hline 'Halilibrahim' & 48.53 & $66.45 \pm 1.71 \mathrm{ab}$ & $46.76 \pm 1.97 \mathrm{c}-\mathrm{f}$ & $7.96 \pm 0.17 \mathrm{fgh}$ & $2.25 \pm 0.30 \mathrm{~b}$ & $2.96 \pm 0.21 \mathrm{bcd}$ \\
\hline 'İnegölkestanesi’ & 53.96 & $65.99 \pm 1.08 \mathrm{ab}$ & $40.99 \pm 0.84 \mathrm{fg}$ & $7.23 \pm 0.16_{1}$ & $1.71 \pm 0.06 \mathrm{c}$ & $2.99 \pm 0.43 \mathrm{bcd}$ \\
\hline ‘İzmitli’ & 47.97 & $63.60 \pm 1.82 \mathrm{abc}$ & $46.12 \pm 0.86 c-f$ & $9.80 \pm 0.41 \mathrm{~d}$ & $1.32 \pm 0.40 \mathrm{f}$ & $3.42 \pm 0.41 \mathrm{bc}$ \\
\hline 'Karamehmet' & 49.95 & $64.36 \pm 0.18 \mathrm{abc}$ & $46.59 \pm 0.70 c-f$ & $8.23 \pm 0.40 \mathrm{efg}$ & $1.73 \pm 0.07 \mathrm{c}$ & $2.79 \pm 0.20 \mathrm{cde}$ \\
\hline 'Korucu’' & 54.71 & $63.57 \pm 2.29 \mathrm{abc}$ & $47.38 \pm 2.88 \mathrm{~b}-\mathrm{e}$ & $8.59 \pm 0.08$ ef & $1.40 \pm 0.17 \mathrm{f}$ & $3.05 \pm 0.21 \mathrm{bcd}$ \\
\hline 'Maraval' & 50.71 & $65.26 \pm 3.61 \mathrm{abc}$ & $46.22 \pm 4.62 c-f$ & $6.45 \pm 0.18 j$ & $1.15 \pm 0.12 \mathrm{~g}$ & $3.43 \pm 0.30 \mathrm{bc}$ \\
\hline 'Marigoule' & 59.47 & $63.76 \pm 10.66 \mathrm{abc}$ & $47.53 \pm 4.51 \mathrm{~b}-\mathrm{e}$ & $8.88 \pm 0.13 \mathrm{e}$ & $0.87 \pm 0.07 \mathrm{~h}$ & $3.56 \pm 0.15 b$ \\
\hline 'Osmanoğlu' & 55.78 & $66.03 \pm 6.54 \mathrm{ab}$ & $45.35 \pm 4.52 \mathrm{def}$ & $7.98 \pm 0.28 \mathrm{fgh}$ & $2.61 \pm 0.20 \mathrm{a}$ & $2.94 \pm 0.85 \mathrm{bcd}$ \\
\hline ‘Öküzgözü’ & 58.05 & $65.53 \pm 1.70 \mathrm{abc}$ & $46.20 \pm 2.25 \mathrm{c}-\mathrm{f}$ & $7.77 \pm 0.19 \mathrm{hg}_{1}$ & $1.54 \pm 0.40 \mathrm{e}$ & $2.89 \pm 0.17 \mathrm{cde}$ \\
\hline 'Sarıaşlama' & 58.52 & $67.13 \pm 1.23 \mathrm{ab}$ & $46.15 \pm 0.95 c-f$ & $10.46 \pm 0.24 c$ & $1.50 \pm 0.16 \mathrm{e}$ & $4.16 \pm 0.21 \mathrm{a}$ \\
\hline 'Sarıkestane' & 48.10 & $67.50 \pm 6.52 \mathrm{ab}$ & $48.23 \pm 3.83 \mathrm{a}-\mathrm{e}$ & $11.22 \pm 0.19 b$ & $1.58 \pm 0.08 \mathrm{de}$ & $2.13 \pm 0.21 \mathrm{f}$ \\
\hline 'Tepeköysarısı' & 46.52 & $60.97 \pm 1.85 b c$ & $37.82 \pm 1.53 \mathrm{~g}$ & $6.15 \pm 0.09 j$ & $0.93 \pm 0.04 \mathrm{~h}$ & $2.27 \pm 0.09 \mathrm{ef}$ \\
\hline
\end{tabular}

Different letters within the same column indicate significant differences (Duncan test, $\mathrm{p}<0.05$ ) 
Table 2. Total sugar, invert sugar and sugar composition of the nineteen chestnut cultivars (mean \pm SD)

\begin{tabular}{|c|c|c|c|c|c|}
\hline Cultivar & $\begin{array}{l}\text { Total sugar } \\
\left(\mathrm{g} / 100 \mathrm{~g}^{-1}\right)\end{array}$ & $\begin{array}{l}\text { Invert sugar } \\
\left(\mathrm{g} / 100 \mathrm{~g}^{-1}\right)\end{array}$ & $\begin{array}{c}\text { Sucrose } \\
\left(\mathrm{g} / 100 \mathrm{~g}^{-1}\right)\end{array}$ & $\begin{array}{l}\text { Glucose } \\
\left(\mathrm{g} / 100 \mathrm{~g}^{-1}\right)\end{array}$ & $\begin{array}{l}\text { Fructose } \\
\left(\mathrm{g} / 100 \mathrm{~g}^{-1}\right)\end{array}$ \\
\hline 'Akkozak' & $12.90 \pm 1.42 \mathrm{~h} 1$ & $2.71 \pm 0.11 \mathrm{~cd}$ & $9.67 \pm 0.77 \mathrm{o}$ & $0.42 \pm 0.01 j$ & $0.45 \pm 0.00 \mathrm{f}$ \\
\hline 'Alimolla' & $11.82 \pm 0.13 \mathrm{ij}$ & $3.39 \pm 0.06 \mathrm{a}$ & $10.78 \pm 0.08 \mathrm{~m}$ & $0.58 \pm 0.00 \mathrm{fg}$ & $0.16 \pm 0.00 \mathrm{k}$ \\
\hline 'Demirci' & $13.55 \pm 0.53 \mathrm{~cd}$ & $3.08 \pm 0.16 \mathrm{abc}$ & $11.59 \pm 0.04 \mathrm{k}$ & $0.43 \pm 0.03 \mathrm{ij}$ & $0.47 \pm 0.03$ ef \\
\hline 'Dursun' & $13.84 \pm 0.36 \mathrm{~h}$ & $2.90 \pm 0.03 \mathrm{a}-\mathrm{d}$ & $10.39 \pm 0.01 \mathrm{n}$ & $0.66 \pm 0.00 \mathrm{de}$ & $0.28 \pm 0.01 \mathrm{ij}$ \\
\hline 'Hacribiş' & $14.15 \pm 1.08 \mathrm{~h}$ & $3.28 \pm 0.10 \mathrm{ab}$ & $13.05 \pm 0.01 \mathrm{~g}$ & $0.79 \pm 0.01 \mathrm{c}$ & $0.37 \pm 0.02 \mathrm{hg}$ \\
\hline 'Hacıömer' & $13.45 \pm 0.72 \mathrm{~h} 1$ & $3.13 \pm 0.47 \mathrm{abc}$ & $12.04 \pm 0.03 \mathrm{j}$ & $0.55 \pm 0.00 \mathrm{gh}$ & $0.57 \pm 0.00 \mathrm{c}$ \\
\hline 'Halilibrahim' & $16.71 \pm 0.47 \mathrm{def}$ & $2.98 \pm 0.13 \mathrm{abc}$ & $13.03 \pm 0.08 \mathrm{~g}$ & $0.75 \pm 0.04 c$ & $0.37 \pm 0.02 \mathrm{gh}$ \\
\hline 'Firdola' & $17.35 \pm 0.34 \mathrm{def}$ & $2.77 \pm 0.12 \mathrm{bd}$ & $15.79 \pm 0.01 \mathrm{~d}$ & $0.64 \pm 0.02$ ef & $0.32 \pm 0.01 \mathrm{~h}_{1}$ \\
\hline 'İnegölkestanesi' & $22.38 \pm 0.30 a$ & $2.62 \pm 0.05 \mathrm{~cd}$ & $16.39 \pm 0.04 c$ & $0.59 \pm 0.04 \mathrm{fg}$ & $0.68 \pm 0.04 b$ \\
\hline 'İzmitli’ & $14.54 \pm 1.04 \mathrm{gh}$ & $2.94 \pm 0.05 \mathrm{a}-\mathrm{d}$ & $11.01 \pm 0.151$ & $0.77 \pm 0.04 c$ & $0.45 \pm 0.01 \mathrm{f}$ \\
\hline 'Karamehmet' & $14.79 \pm 0.56$ & $2.97 \pm 0.04 \mathrm{abc}$ & $12.45 \pm 0.031$ & $0.72 \pm 0.02 \mathrm{~cd}$ & $0.29 \pm 0.01 \mathrm{ij}$ \\
\hline 'Korucu’' & $13.17 \pm 0.78 \mathrm{~h}_{1}$ & $3.02 \pm 0.18 \mathrm{abc}$ & $9.63 \pm 0.09$ o & $0.41 \pm 0.01 \mathrm{j}$ & $0.41 \pm 0.01 \mathrm{fg}$ \\
\hline 'Maraval' & $16.09 \pm 1.30 \mathrm{fg}$ & $2.95 \pm 0.29 \mathrm{a}-\mathrm{d}$ & $12.69 \pm 0.04 \mathrm{~h}$ & $0.62 \pm 0.00 \mathrm{efg}$ & $0.15 \pm 0.01 \mathrm{k}$ \\
\hline 'Marigoule' & $17.03 \pm 0.78 \mathrm{def}$ & $2.80 \pm 0.68 \mathrm{bcd}$ & $14.10 \pm 0.09 \mathrm{f}$ & $0.34 \pm 0.02 \mathrm{k}$ & $0.23 \pm 0.01 j$ \\
\hline 'Osmanoğlu' & $19.49 \pm 1.26 \mathrm{bc}$ & $2.79 \pm 0.47 \mathrm{bcd}$ & $15.41 \pm 0.06 \mathrm{e}$ & $1.13 \pm 0.09 \mathrm{a}$ & $0.51 \pm 0.04 \mathrm{de}$ \\
\hline ‘Öküzgözü’ & $16.38 \pm 1.24 \mathrm{eg}$ & $2.95 \pm 0.14 \mathrm{a}-\mathrm{d}$ & $12.04 \pm 0.13 j$ & $0.49 \pm 0.01 \mathrm{~h} 1$ & $0.54 \pm 0.02 \mathrm{~cd}$ \\
\hline 'Sarıaşlama' & $18.04 \pm 1.75 \mathrm{cde}$ & $2.95 \pm 0.06 \mathrm{a}-\mathrm{d}$ & $16.63 \pm 0.04 b$ & $0.94 \pm 0.04 b$ & $0.80 \pm 0.05 \mathrm{a}$ \\
\hline 'Sarıkestane' & $10.59 \pm 2.17 j$ & $3.41 \pm 0.52 \mathrm{a}$ & $6.82 \pm 0.10 \mathrm{p}$ & $0.40 \pm 0.00 \mathrm{j}$ & $0.23 \pm 0.00 \mathrm{j}$ \\
\hline 'Tepeköysarısı' & $20.74 \pm 0.23 \mathrm{ab}$ & $2.41 \pm 0.10 \mathrm{de}$ & $17.40 \pm 0.07 a$ & $1.00 \pm 0.04 b$ & $0.65 \pm 0.02 b$ \\
\hline
\end{tabular}

Different letters within the same column indicate significant differences (Duncan test, $\mathrm{p}<0.05$ )

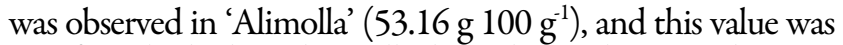
significantly higher than all the other cultivars. This was followed by 'Demirci' ( $52.90 \mathrm{~g} / 100 \mathrm{~g}$ ) and 'Haciibiş' ( $51.45 \mathrm{~g}$ $\left.100 \mathrm{~g}^{-1}\right)$. The lowest starch content was recorded in

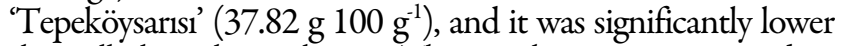
than all the other cultivars. The starch content reported in Chinese chestnuts ranged from 38.6 to $62.8 \%$ (Yang et al., 2015), from 38.6 to $47.9 \%$ in chestnut cultivars from Portugal (Borges et al., 2008), from 42.2 to $66.5 \%$ in chestnut cultivars from Spain (Pereira-Lorenzo et al., 2006), and from 40.85 to $56.32 \mathrm{~g} / 100 \mathrm{~g}^{-1}$ in chestnut cultivars from Bulgaria (Glushkova et al., 2010).

Protein content varied significantly among cultivars $(\mathrm{p}<$ 0.05 ). The protein content of the chestnuts ranged from 6.15 (cv. 'Tepeköysarısi') to $12.44 \mathrm{~g} 100 \mathrm{~g}^{-1}$ (cv. 'Dursun'), which

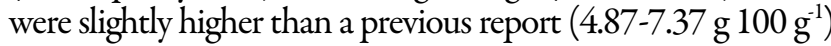
(Ertürk et al. 2006). The protein content was reported to be

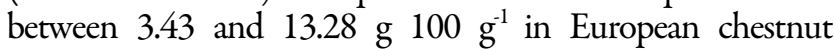
cultivars and between $6.1 \%$ and $12.2 \%$ in Chinese chestnut cultivars (Ferreria-Cardoso et al., 1993; Pinnavaia et al., 1993; Brighenti et al., 1998; Bounous, 1999; Ertürk et al., 2006; Borges et al., 2008; Yang et al., 2015). Our results are similar to previous results.

Chestnuts differ from other nuts and have lower fat (2-5\%) content. The crude fat content was significantly different among the cultivars $(\mathrm{p}<0.05)$. The crude fat amount of all the cultivars ranged from 0.87 to $2.61 \mathrm{~g} 100 \mathrm{~g}^{-1}$ for cvs. 'Marigoule' and 'Osmanoğlu', respectively. The results are in accordance with those obtained by Ertürk et al. (2006) for C. sativa and hybrid cultivars, (Sacchetti and Pinnavaia, 2005) for Italian cultivars and De La Montana Miguelez et al. (2004) and Pereira-Lorenzo et al. (2006) for Spanish cultivars.

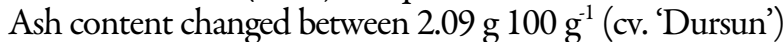

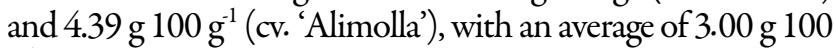
$\mathrm{g}^{-1}$ (Table 1). The quantity of ash reported in chestnut ranged between 0.83 and $4.92 \mathrm{~g} 100 \mathrm{~g}^{-1}$ in various species and genotypes (Brighenti et al., 1998; Demiate et al., 2001; Sundriyal and Sundriyal, 2001; Ertürk et al., 2006; Pereira-
Lorenzo et al., 2006; Bourges et al., 2008; Sacchetti et al., 2009). Our results were similar to previous results.

Invert and total sugars varied significantly among the cultivars $(p<0.05)$. The invert sugar content of the cultivars

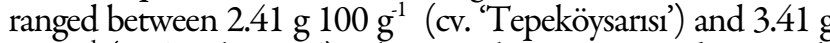
$100 \mathrm{~g}^{-1}$ (cv. 'Sarıkestane'). These results are in accordance with Pinnavaia et al. (1993) for French cultivars (0.82 and $3.56 \mathrm{~g}$

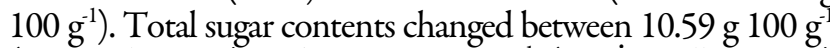

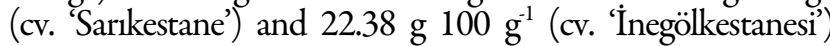
(Table 2). Within the range, the results of Pinnavaia et al. (1993), Bounous et al. (2000) and Ertürk et al. (2006) reported

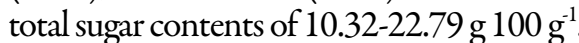

Sucrose is the main sugar in the chestnuts. Chestnut cultivars and hybrids showed heterogeneity with respect to their sugar contents. Significant differences $(p>0.05)$ were found among the cultivars for sucrose, glucose and fructose contents (Table 2). The sucrose, glucose and fructose contents

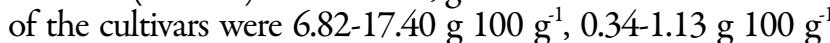
and $0.15-0.80 \mathrm{~g} 100 \mathrm{~g}^{-1}$, respectively. The 'Tepeköysarıs'

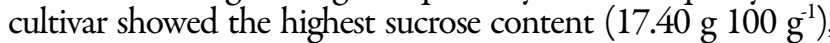
while the 'Sarıkestane' cultivar had the lowest value $(6.82 \mathrm{~g} 100$ $\left.\mathrm{g}^{-1}\right)$. Glucose and fructose were also present in lower quantities in the chestnut samples (Table 2). Bernárdez et al. (2004) found slightly higher concentrations of sucrose $(6.5$ and $19.5 \mathrm{~g}$ $\left.100 \mathrm{~g}^{-1}\right)$ and lower concentrations of glucose $(0.00$ and $0.27 \mathrm{~g}$

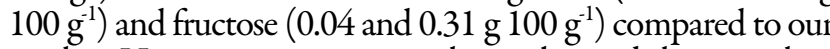
results. However, some researchers observed lower values

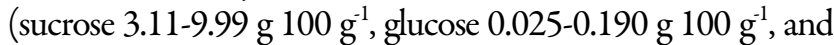

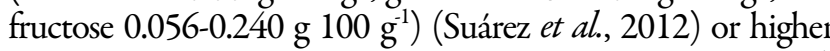
values (Barreira et al., 2010) (sucrose 9.56-22.05 g $100 \mathrm{~g}^{-1}$,

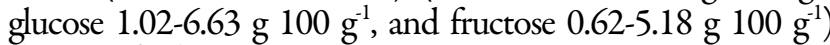
than our findings.

Table 3 shows the coefficients of variation in the chemical parameters of the chestnut seeds from the different cultivars in order to verify the intravarietal homogeneity. Chestnuts from cvs. 'Marigoule' 'Sarıkestane', 'Osmanoğlu', 'Haciömer', 'Alimolla' and 'Demirci' showed just one coefficient of variation higher than $15 \%$ in carbohydrates, total sugar, invert sugar, starch, crude protein and ash parameters. The 
Table 3. Coefficients of variation $\left[(\mathrm{SD} / \mathrm{mean})^{*} 100\right]$ of main parameters analysed

\begin{tabular}{cccccccccccc}
\hline Cultivar & Carbohydrates & $\begin{array}{c}\text { Total } \\
\text { sugar }\end{array}$ & $\begin{array}{c}\text { Invert } \\
\text { sugar }\end{array}$ & Starch & $\begin{array}{c}\text { Crude } \\
\text { protein }\end{array}$ & $\begin{array}{c}\text { Crude } \\
\text { fat }\end{array}$ & Ash & Sucrose & Glucose & Fructose \\
\hline 'Akkozak' & 0.85 & 11.01 & 4.22 & 4.22 & 4.50 & 2.94 & 0.44 & 7.96 & 3.36 & 0.00 \\
'Alimolla' & 1.61 & 1.09 & 1.75 & 1.75 & 15.68 & 3.43 & 9.82 & 0.72 & 0.00 & 0.00 \\
'Demirci' & 4.58 & 3.90 & 5.19 & 4.79 & 1.89 & 1.93 & 16.24 & 0.35 & 6.58 & 6.02 \\
'Dursun' & 1.27 & 2.56 & 1.09 & 1.09 & 2.40 & 2.02 & 4.71 & 0.10 & 0.00 & 2.57 \\
'Hacibisiş' & 1.16 & 7.64 & 3.18 & 3.18 & 5.84 & 1.79 & 7.91 & 0.08 & 0.90 & 5.81 \\
'Haciömer' & 11.21 & 5.34 & 14.96 & 14.96 & 4.82 & 3.08 & 16.55 & 0.25 & 0.00 & 0.00 \\
'Halilibrahim' & 2.57 & 2.83 & 4.21 & 4.21 & 2.09 & 0.63 & 6.98 & 0.61 & 4.75 & 5.81 \\
'Firdola' & 3.20 & 1.98 & 4.20 & 4.20 & 3.41 & 2.72 & 1.90 & 0.09 & 3.34 & 2.24 \\
'Inegölkestanesi' & 1.64 & 1.32 & 2.05 & 2.05 & 2.16 & 3.31 & 14.44 & 0.26 & 6.05 & 5.24 \\
'İzmitli' & 2.87 & 7.15 & 1.86 & 1.86 & 4.16 & 2.70 & 12.08 & 1.36 & 5.51 & 1.59 \\
'Karamehmet' & 0.28 & 3.81 & 1.49 & 1.49 & 4.81 & 4.09 & 7.19 & 0.22 & 2.97 & 2.48 \\
'Korucu' & 3.60 & 5.89 & 6.07 & 6.07 & 0.91 & 2.02 & 6.77 & 0.93 & 3.44 & 3.45 \\
'Maraval' & 5.54 & 8.07 & 9.99 & 9.99 & 2.76 & 1.85 & 8.62 & 0.28 & 0.00 & 9.43 \\
'Marigoule' & 16.72 & 4.57 & 24.39 & 9.50 & 1.51 & 8.13 & 4.16 & 0.65 & 6.33 & 3.14 \\
'Osmanoğlu' & 9.91 & 6.44 & 16.69 & 9.98 & 3.51 & 2.17 & 28.92 & 0.42 & 8.17 & 7.84 \\
'Öküzgözü' & 2.60 & 7.57 & 4.88 & 4.88 & 2.44 & 1.84 & 5.88 & 1.11 & 2.88 & 3.96 \\
'Sarraşlama' & 1.83 & 9.67 & 2.06 & 2.06 & 2.26 & 0.94 & 5.08 & 0.25 & 4.51 & 6.23 \\
'Sarkestane' & 9.66 & 20.51 & 15.12 & 16.77 & 1.67 & 5.37 & 9.89 & 1.47 & 0.00 & 0.00 \\
'Tepeköysarısi & 3.03 & 1.09 & 4.04 & 4.04 & 1.45 & 4.56 & 3.96 & 0.40 & 3.56 & 3.29 \\
\hline
\end{tabular}
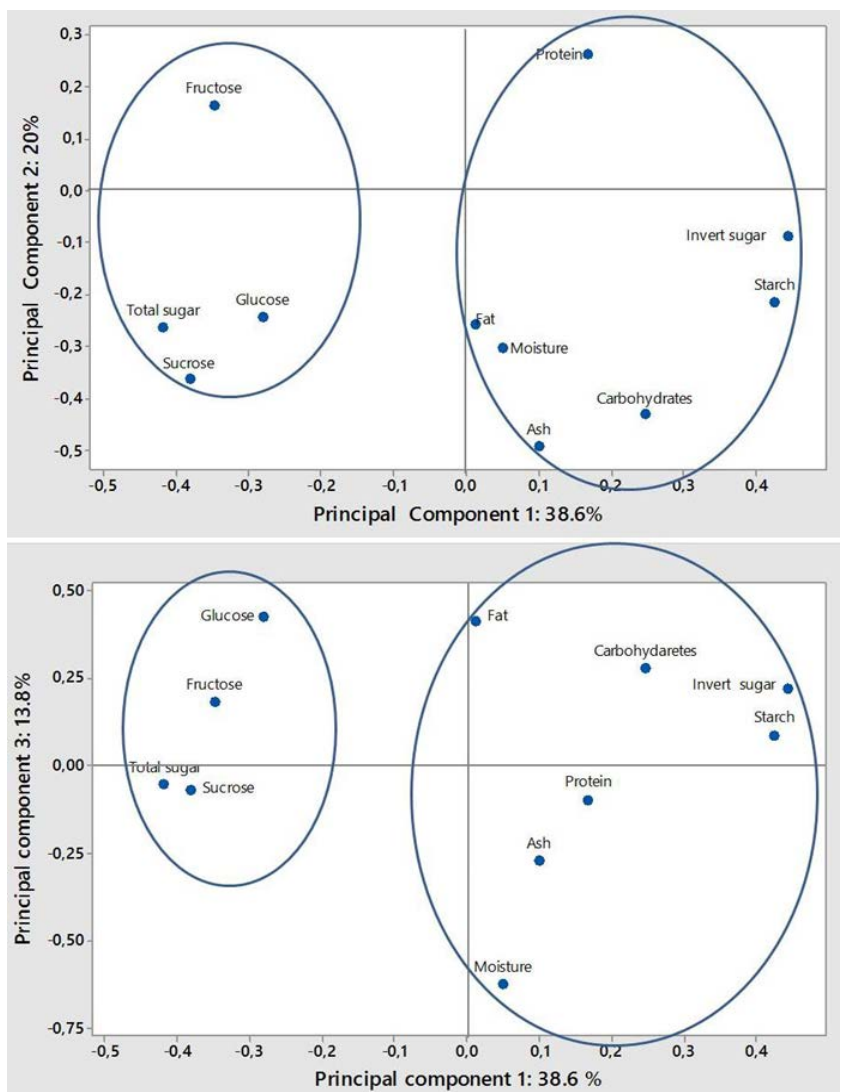

Fig. 1. Principal component analysis plot for nutritional parameters of the nineteen chestnut cultivars

coefficients of variation of the other chestnut cultivars were lower than $12 \%$, distinguishing their homogeneity. PCA was applied to moisture, carbohydrates, total sugars, crude protein, crude fat, starch, invert sugars, ash, sucrose, fructose, glucose for the nineteen cultivars (Fig. 1). The results from the PCA showed that the first three principal components (PC1, PC2 and PC3) accounted for $72.4 \%$ of the variation (Fig. 1). PC1 explained $38.6 \%$ of the TV, and the parameters that correlated best with this PC were invert sugar $(0.444)$, starch $(0.425)$ and total sugar $(-0.418)$. The parameters that best correlated with
PC2 (that accounted for $20 \%$ of TV) were ash $(-0.493)$, carbohydrates $(-0.432)$ and sucrose $(-0.364)$, and with PC3 (that accounted for $13.8 \%$ of TV), the parameters were moisture (-0.626), glucose $(0.425)$ and crude fat $(0.414)$. Two groups were defined according to the position of each sample. The total sugar, sucrose, glucose, fructose showed similar variation in relation to the PC1, whilst starch, protein, ash, moisture, invert sugar, carbohydrates, and crude fat showed an inverse variation.

\section{Conclusions}

The results obtained in this study do confirm that chestnuts are a rich source of carbohydrate and starch. In addition to sucrose, the predominant free sugars, glucose, and fructose also existed in our studied chestnuts. The results showed that, the chemical composition of studied cultivars varied depending on genetic structure and ecological conditions. Differences of nutritional value and other quality parameters among cultivars could be very useful in selecting cultivars for different purpose. For instance, 'Alimolla', 'Demirci' and 'Hacibisis' cultivars are appropriate for flour production due to their high starch content. 'Inegölkestanesi', 'Tepeköysarıs' and 'Osmanoğlu' with high quantities of total sugar are more desirable for fresh consumption. The results of this study will be a benefit to producers, consumers, breeders and the processing industry.

\section{References}

AOAC (2000). Official methods of analysis (17th Ed). Washington, DC: Association of Official Analytical Chemists.

Barreira JCM, Pereira JA, Oliveira MBPP, Ferreira ICFR (2010). Sugars profiles of different chestnut (Castaneasativa Mill). and almond (Prumus dulis) cultivars by HPLC-RI. Plant Foods for Human Nutrition 65:3843.

Bernárdez MM, Miguelez JM, Queijeiro JG (2004) HPLC determination of sugars in varieties of chestnut fruits from Galicia (Spain). Journal of Food Composition and Analysis 17:63-67. 
Bounous G (1999). Among the chestnut trees in Cuneo province. Edizioni Metaforevia Carlo Emanuele, Cuneo, Italy.

Bounous G, Botta R, Beccaro G (2000). The chestnut: the ultimate energy source nutritional value and alimentary benefits. Nucis 9:4450.

Borges O, Gonçalves B, de Carvalho JLS, Correia P, Silva AP (2008). Nutritional quality of chestnut (Castanea sativa Mill.) cultivars from Portugal. Food Chemistry 106:976-984.

Brighenti F, Campagnolo M, Bassi D (1998). Biochemical characterization of the seed in instinct chestnut genotypes (C. sativa). In: Abstracts Book, 2nd International Symposium on Chestnut. Bordeaux, France.

Correia P, Leitao A, Beirao-da-Costa ML (2009). The effect of drying temperatures on morphological and chemical properties of dried chestnuts flours. Journal of Food Engineering 90:325-332.

De La Montana Miguelez J, Bernárdez MM, Garcia Queijeiro JM (2004). Composition of varieties of chestnuts from Galicia (Spain). Food Chemistry 84(3):401-404.

Demiate IM, Oetterer M, Wosiacki G (2001). Characterization of chestnut (Castanea sativa Mill) starch for industrial utilization. Brazilian Archives of Biology and Technology 44(1):69-78.

Ertan E (2007). Variability in leaf and fruit morphology and in fruit composition of chestnuts (Castanea sativa Mill.) in the Nazilli region of Turkey. Genetic Resources and Crop Evolution 54:691-699.

Ertürk U, Mert C, Soylu A (2006). Chemical composition of fruits of some important chestnut cultivars. Brazilian Archives of Biology and Technology 49:183-188.

Ferreria-Cardoso JV, Fontainhas-Fernandes AA, Torres-Pereira JMG (1993). Nutritive value and technological characteristics of Castanea sativa Mill. fruits - comparative study of some Northeastern Portugal cultivars. In: Proceedings of the 1st International Congress on Chestnut. Spoleto, Italypp 445-449.

Glushkova M, Zhyanski M, Velinova K (2010). Nut quality assessment of chestnut cultivars from 'Ivanik' clone collection. Forest Science 1:3-14.

Künsch U, Scharer H, Conedera M, Sassella A, Jermini M, Jelmini G (1999). Quality assessment of chestnut fruits. Acta Horticulturae 494:119-128.

McCarthy MA, Meredith FI (1988). Nutrient data on chestnuts consumed in the United States. Economic Botany 42:29-36.

Mujić I, Agayn V, ŽivkovićJ, Velić D, JokićS, Alibabić V, Rekić A (2010). Chestnuts, a "Comfort" Healthy Food?. Acta Horticulturae 866:659665.

Neri L, Dimitri G, Sacchetti G (2010). Chemical composition and antioxidant activity of cured chestnuts from three sweet chestnut (Castanea sativa Mill.) ecotypes from Italy. The Journal of Food Composition and Analysis 23:23-29.
Pereira-Lorenzo S, Ramos-Cabrer AM, Diaz-Hernández MB, Ciordia-Ara M, Rios-Mesa D (2006). Chemical composition of chestnut cultivars from Spain. Scientia Horticulturae 107(3):306-314.

Pinnavaia GG, Pizzirani S, Severini C, Bassi D (1993). Chemical and functional characterization of some chestnut varieties. In: Proceedings of the 1st International Congress on Chestnut. Spoleto, Italy pp 319-326.

Poljak I, Vahčić N, Gačić M, Idžojtić M (2016). Morphological characterization and chemical composition of fruits of the traditional Croatian chestnut variety 'lovran marron'. Food Technology and Biotechnology 54(2):189-199.

Ross FA (1959). Dinitrophenol methods for reducing sugars. In: Talburt WF, Smith O (Eds). Potato Processing. A VI Publishing Comp. Connecticutpp 469-470.

Sacchetti G, Pinnavaia GG (2005). Compositional characteristics of some chestnut biotypes of Emiliano-Romagnolo Apennine. Acta Horticulturae 693:241-246.

Sacchetti G, Neri L, Dimitri G, Mastrocola D (2009). Chemical composition and functional properties of three sweet chestnut (Castanea sativa Mill.) ecotypes from Italy. Acta Horticulturae 844:4146.

Silvanini A, Dall'Asta C, Morrone L, Cirlini M, Beghe D, Fabbri A, Ganino $\mathrm{T}$ (2014). Altitude effects on fruit morphology and flour composition of two chestnut cultivars. Scientia Horticulturae 176:311-318.

Soylu A, Mert C (2009). The future of chestnut breeding work in Turkey. Acta Horticulturae 815:27-32.

Soylu A, Serdar U, Ertan E, Mert C (2009). Turkey. In: Avanzato D (Ed). Following chestnut footprints (Castanea spp.) - Cultivation and culture, folklore and history, traditions and uses. Scripta Horticulturae No 9, ISHS Press, Leuven, Belgium pp155-160.

Suárez MH, Galdón BR, Mesa DR, Romero CD, Rodríguez ER (2012). Sugars, organic acids and total phenols in varieties of chestnut fruits from Tenerife(Spain). Food and Nutrition Sciences 3:705-715.

Sundriyal M, Sundriyal RC (2001). Wild edible plants of the Sikkim Himalaya: nutritive values of selected species. Economic Botany 55(3):377-390.

Uylaser V, Yildız G, Mert C, Serdar U. (2014). A general assessment of the candied chestnut industry in Turkey. Acta Horticulturae 1019:229234.

Warmund MR, Elmore JR, Adhikari K, McGraw S (2011). Descriptive sensory analysis and free sugar contents of chestnut cultivars grown in North America. Journal of the Science of Food Agriculture 91:19401945.

Yang F, Liu Q, Pan S, Xu C, Xiong YL (2015). Chemical composition and quality traits of Chinese chestnuts (Castanea mollissima) produced in different ecological regions. Food Bioscience 11:33-42. 